\title{
SETS WITH LARGE LOCAL INDEX OF QUASIREGULAR MAPPINGS IN DIMENSION THREE
}

\author{
SEPPO RICKMAN
}

\section{Introduction}

Interesting quasiregular mappings have usually nonempty branch set if the dimension is greater than two. This is perhaps best illustrated by Zorič's theorem [7] which says that a locally homeomorphic quasiregular mapping of the Euclidean $n$-space $R^{n}$ into itself is in fact a homeomorphism if $n \geqq 3$. For a nonconstant quasiregular mapping $f: G \rightarrow R^{n}$ the local index at $x$ is $i(x, f)=\sup _{y}$ card $U \cap f^{-1}(y)$ where $U$ is any sufficiently small neighborhood of $x$. For the basic theory of quasiregular mappings, see [2]. Although the branch set $B_{f}$ is often nonempty for a quasiregular mapping $f$, the local index cannot be too large in the whole branch set if $n \geqq 3$. Results in this direction were first proved by Martio in [1]. One of his main results $[1,6.8]$ is that

$$
\inf _{x \in F} i(x, f)<K_{I}(f)\left(\frac{n}{p}\right)^{n-1}
$$

for any compact set $F \subset B_{f}$ with $\mathscr{H}^{p}(f F)>0$ where $\mathscr{H}^{p}$ is the $p$-dimensional Hausdorff measure. Here $K_{I}(f)$ is the inner dilatation defined as the smallest $K$ satisfying

$$
J_{f}(x) \leqq K \inf _{|h|=1}\left|f^{\prime}(x) h\right|^{n} \quad \text { a.e. }
$$

In $[3,3.4]$ it was proved that $\mathscr{H}^{n-2}\left(f B_{f}\right)>0$ if $B_{f} \neq \emptyset$. Then $F$ can be chosen so that (1.1) holds for $p=n-2$. Applying (1.1) to continua $F$ we can also deduce that the set $\left\{x \in G \mid\left(i(x, f) / K_{I}(f)\right)^{1 /(n-1)}>n\right\}$ is totally disconnected. This follows also from Theorem 1.2 below.

An example of a nonconstant quasiregular mapping $f: G \rightarrow R^{n}$ with $\sup \{i(x, f) \mid x \in G\}=\infty$ was given in $[3,4.10]$. The set $E_{c}=\{x \in G \mid i(x, f) \geqq c\}$, which is closed in $G$, has in that example no accumulation points in $G$ for large $c$. It has been conjectured that this is always the case for some $c=c(n, K)$ for any $K$-quasiregular mapping. In [6] we proved the following result which shows that if there is some even distribution among points $x_{0}, \ldots, x_{m}$ with $m$ sufficiently large, then the local index cannot maintain a high constant value at these points. 
1.2. Theorem [6]. Let $f: G \rightarrow R^{n}$ be nonconstant and quasiregular. For each point $x_{0}$ there exist positive numbers $t_{0}$ and $p_{0}$ such that the following holds. If $1 \leqq v<$ $\mu=\left(i\left(x_{0}, f\right) / K_{I}(f)\right)^{1 /(n-1)}$ and if $x_{0}, \ldots, x_{m}$ are points in the ball $\bar{B}^{n}\left(x_{0}, t\right)$ such that $\left|x_{j}-x_{j+1}\right| \leqq t / p,\left|x_{0}-x_{m}\right|=t$, and $m \leqq p^{v}, p \geqq p_{0}, t \leqq t_{0}$, then there exists $j \in\{1, \ldots, m\}$ with $i\left(x_{j}, f\right)<i\left(x_{0}, f\right)$.

The purpose of this paper is to show that the conjecture above is false for dimension three. In fact we are able to prove the following result.

1.3. Theorem. There exists $K>1$ such that for each $c>0$ there exists a $K$ quasimeromorphic mapping $h: \bar{R}^{3} \rightarrow \bar{R}^{3}$ with $E_{c}=\left\{x \in \bar{R}^{3} \mid \mu(h)=i(x, h) \geqq c\right\}$ a Cantor set. Here $\mu(h)$ is the degree of $h$.

Theorem 1.2 shows that the set $E_{c}$ in 1.3 cannot be evenly distributed for sufficiently large values of $c$. The proof of 1.3 depends on the construction in [5] where it is shown that there exists a nonconstant quasiregular mapping of $R^{3}$ into itself omitting any prescribed finite number of points. It can be shown that such a mapping must be of complicated nature. I believe that a map $h$ like in Theorem 1.3 must also be complicated for large $c$. Whether the result in [5] holds for dimensions $n \geqq 4$, is an open question. Consequently, also 1.3 is an open question for $n \geqq 4$.

Quasiregular mappings form the right extension of the theory of analytic functions in the plane to real $n$-dimensional space. Surprisingly strong results are true even for value distribution of these mappings. For a defect relation, see [4]. In the classical theory there is a direct connection between branching and covering, which in the simple case of a nonconstant rational function $f: \bar{R}^{2} \rightarrow \bar{R}^{2}$ is presented by the Hurwitz formula

$$
\sum_{x \in \bar{R}^{2}}(i(x, f)-1)=2 \mu(f)-2 .
$$

It has been asked whether there exists a connection of this type also in higher dimensions, for example in the form

$$
\sum_{x \in R^{n}}(i(x, f)-c(n, K))_{+} \leqq M(n, K) \mu(f)
$$

for a nonconstant $K$-quasimeromorphic mapping $f: \bar{R}^{n} \rightarrow \bar{R}^{n}$. Theorem 1.3 gives a negative answer to this in dimension three.

It was proved in $[1,6.5]$ that

$$
\inf _{x \in \boldsymbol{F}} i(x, f) \leqq K_{I}(f)
$$

if $F$ is a rectifiable arc in $B_{f}$ and $f$ is a nonconstant quasiregular mapping. Since the left hand side is always at least 2 , we obtain in this case $K_{I}(f) \geqq 2$. For the mapping $f: R^{3} \rightarrow R^{3}, f\left(r, \varphi, x_{3}\right)=\left(r, 2 \varphi, x_{3}\right)$ in cylindrical coordinates, $K_{I}(f)=2$, and also for a similar "winding map" in higher dimensions. It is an interesting open question whether a quasiregular mapping $f$ with $K_{I}(f)<2$ must always have an empty branch 
set. The branch set need not contain any rectifiable arc, see [3, 4.7]. It is known [3, 4.6] that for each dimension $n$ there exists $K_{n}>1$ such that $B_{f}=\emptyset$ for every $K_{n}$-quasiregular mapping $f: G \rightarrow R^{n}$. Note that for the extreme case $v=1$ in Theorem 1.2 the situation $K_{I}(f)<i\left(x_{0}, f\right)=2$ also applies.

\section{Background for the construction}

The proof of Theorem 1.3 is based almost completely on the constructions in [5]. We shall therefore make full advantage of the notation and results in [5]. The idea is roughly the following. With some minor modification we shall take the construction for a quasiregular map of $R^{3}$ omitting $p=2$ points in $R^{3}$ and consider the restriction to a certain compact part $A$ which is homeomorphic to a ball. We glue together a similar restriction, this time defined in a set $A^{\prime}$ with no common interior points with $A$ and such that the complement of $A \cup A^{\prime}$ consists of three topological balls $B_{1}, B_{2}$, $B_{3}$. We are able to extend the mapping to these topological balls such that in each $B_{j}$ there exists a point $x_{j}$ at which the local index agrees with the degree of the resulting map. This process can be repeated, and each ball $B_{j}$ will then be replaced by two balls in each of which the new map has a point with local index equal to the degree, etc.

Following now [5] let $u_{1}=\infty, u_{2}=-e_{3} / 2, u_{3}=e_{3} / 2$ and let $U_{1}, U_{2}, U_{3}$ be the components of $R^{3} \backslash\left(S^{2} \cup B^{2} \cup\left\{u_{2}, u_{3}\right\}\right)$ such that $u_{j} \in \bar{U}_{j}, j=2,3$. The constructed map $f: R^{3} \rightarrow R^{3} \backslash\left\{u_{2}, u_{3}\right\}$ in [5] has the property that $W_{j}=f^{-1} U_{j}, j=1,2$, consists of one component and $W_{3}=f^{-1} U_{3}$ of six components. We shall first work with one component of $W_{3}$, denoted by $W_{3}(0)$ (see the end of $[5,7.2]$ ). We fix a large positive even integer $k$, depending on the value of $c$ in Theorem 1.3. Recall the notions $M_{k, k-1}(0)$, $M_{k, k-1, j}(0)$, and $G_{k, k-1, j}(0), j=1,2,3$, from [5. 4.1]. The sets $M_{k, k-1}(0)$ and $M_{k, k-1, j}(0)$ are certain unions of simplicial 2-complexes and $G_{k, k-1, j}(0)$ is called a map complex. The preimage $f^{-1}\left(S^{2} \cup B^{2}\right)$ is $\left|\mathscr{H}^{2}\right|$ where $\mathscr{H}^{2}$ is the set of elements called sheets [5, 7.2]. A part of $\mathscr{H}^{2}$ is inherited from $M_{k, k-1}(0)$, call it $\mathscr{H}_{k, k-1}^{2}(0)$. We now reflect the objects $M_{k, k-1}(0), M_{k, k-1, j}(0)$, and $G_{k, k-1, j}(0)$ through the plane $T=\left\{x \in R^{3} \mid x_{1}=\sqrt{3} v^{k} / 2\right\}$ and obtain $M_{k, k-1}^{*}(0)$ etc. The construction is done in such a way that $\left|M_{k, k-1}(0) \cup M_{k, k-1}^{*}(0)\right|$ bounds a bounded set $V$ homeomorphic to an open 3-ball. In a similar way as the level surfaces $v^{2 i}\left|N_{3}(0)\right|, i=0,1, \ldots$, were constructed in $V_{3}(0)$ in [5, Section 4], we can for our purpose construct a finite number of level surfaces $\left|N_{1}^{\prime}\right|, \ldots,\left|N_{k / 2}^{\prime}\right|$ in $V$, all homeomorphic to $S^{2}$. The construction of these can be made so that they are symmetric with respect to the plane $T$ and so that $\left|N_{i+1}^{\prime}\right| \cap C$ coincides almost with $v^{2 i}\left|N_{3}(0)\right| \cap C$ where $C$ is the component of the complement of $T$ which contains the origin.

The structure of the set $\mathscr{H}^{2}$ of sheets is determined by a union $\mathscr{G}_{\infty}$ of map complexes defined in [5, 7.1]. Let the part of $\mathscr{G}_{\infty}$ which lies in $\left|M_{k, k-1}(0)\right|$ be $\mathscr{G}_{k, k-1}(0)$. We reflect also $\mathscr{G}_{k, k-1}(0)$ with respect to $T$ and get $\mathscr{G}_{k, k-1}^{*}(0)$. This $\mathscr{G}_{k, k-1}^{*}(0)$ defines then a set $\hat{\mathscr{H}}_{k, k-1}^{2}(0)$ of sheets which are inherited from $M_{k, k-1}^{*}(0)$. We point out 
that $\hat{\mathscr{H}}_{k, k-1}^{2}(0)$ is not obtained from $\mathscr{H}_{k, k-1}^{2}(0)$ by reflection in $T$ because a so called positive (negative) element in $\mathscr{G}_{k, k-1}^{2}$ is taken to a negative (positive) one in $\mathscr{G}_{k, k-1}^{* 2}$ by this reflection, and to a positive (negative) element we attach 2 (4) sheets (see $[5,7.1,7.2])$. The set $Y=\left|\mathscr{H}_{k, k-1}^{2}(0) \cup \hat{\mathscr{H}}_{k, k-1}^{2}(0)\right|$ bounds a bounded set $W$ homeomorphic to an open ball.

Next we are going to construct a quasiregular map $w^{\prime \prime}$ of a subset $W^{\prime \prime}$ of $W$, bounded by $Y$ and $\left|N_{k / 2}^{\prime}\right|$, onto $U_{3} \backslash \bar{B}^{3}\left(u_{3}, r\right)$ with some $r>0$ along the lines of [5]. To apply the various steps in [5] to this case we need to define suitable map complexes $H_{1}^{\prime}, \ldots, H_{k / 2}^{\prime}$ on the level surfaces $\left|N_{1}^{\prime}\right|, \ldots,\left|N_{k / 2}^{\prime}\right|$. In [5] the underlying space of a map complex is always homeomorphic to $R^{2}$ or a closed disk, but the definition extends clearly to this case. These map complexes $H_{1}^{\prime}, \ldots, H_{k / 2}^{\prime}$ will now be finite and $H_{i+1}^{\prime}$ can on $\left|N_{i+1}^{\prime}\right| \cap C$ be almost copied in an obvious way from a corresponding part of the map complex $v^{2 i} \mathrm{H}_{3}(0)$ on $v^{2 i}\left|N_{3}(0)\right|$. Here $H_{3}(0)$ is the map complex on $\left|N_{3}(0)\right|$ corresponding to $H_{1}$ on $\left|N_{1}\right|$ which is defined in [5, 4.3]. We may further require that $H_{i+1}^{\prime}$ is symmetric with respect to the plane $T$. After these preparations we are ready to use the method of Sections 4-7 in [5] to obtain the required map $w^{\prime \prime}: W^{\prime \prime} \rightarrow U_{3} \backslash \bar{B}^{3}\left(u_{3}, r\right)$.

Now we fix a regular (closed) 3-simplex $\Delta^{\prime}$ in $W \backslash W^{\prime \prime}$ with side length $2 a$ and with center $v$ in $T$. Let $\Delta$ be the concentric 3-simplex with side length $a$. On the boundary $\partial \Delta$ we fix a map complex $G$ with $\sigma(G)=\sigma\left(H_{1}^{\prime}\right)$ where $\sigma(G)$ denotes the number of 2-simplexes of $G$. In addition, we may require that there are positive constants $c_{1}$ and $c_{2}$, independent of $k$, such that $c_{1} \leqq \operatorname{diam}(A) \sigma(G)^{1 / 2} / a \leqq c_{2}$ for all 2simplexes $A$ in $G$. Again using the method of Sections 4-7 in [5] we extend $w^{\prime \prime}$ to a map $w^{\prime}: W \backslash$ int $\Delta \rightarrow U_{3} \backslash B^{3}\left(u_{3}, t\right)$ for some $\left.t \in\right] 0, r$ [ which is quasiregular in $W \backslash \Delta$ and $w^{\prime} \mid \partial \Delta: \partial \Delta \rightarrow S^{2}\left(u_{3}, t\right)$ is represented by the map complex $G$ in the sense of $[5,5.1]$ up to a similarity map taking $S^{2}\left(u_{3}, t\right)$ onto $S^{2}$. Now it is a simple matter to extend $w^{\prime}$ radially further to a quasiregular map $w: W \rightarrow$ int $\bar{U}_{3}$ so that each cone $C_{A}=\{v+t(y-v) \mid y \in A, 0 \leqq t \leqq 1\}$, with $A$ a 2 -simplex in $G$, is opened up to a half of $\bar{B}^{3}\left(u_{3}, t\right)$. The local index of $w$ at $v$ will then be $\sigma(G) / 2$.

\section{Proof of Theorem 1.3}

Following [5, 3.1] let $\left|M_{k 0}\right|$ be the 2-simplex $\left\{x \in R^{2}|\sqrt{3}| x_{2} \mid \leqq x_{1} \leqq \sqrt{3} v^{k} / 2\right\}$ and let $\left|M_{k 0}^{*}\right|$ be $\left|M_{k 0}\right|$ reflected with respect to the plane $T$. Let the boundary $\left|\left(M_{k 0} \cup M_{k 0}^{*}\right)\right|$ be $A_{1}$. We shall next perform a quasiconformal map of the domain

$$
D=\left\{x \in R^{3}\left|\quad\left(x_{1}, x_{2}\right) \in\right| M_{k 0}|\cup| M_{k 0}^{*}|, \quad| x_{3} \mid<d\left(x, A_{1}\right) / 2\right\}
$$

where $d$ is the Euclidean distance. The construction of the sheets can be done so that $Y$ lies inside $\bar{D}$. First we map $D$ onto $D^{\prime}=\{\tau x \mid x \in D, \tau>0\}$ by a map $\psi_{1}$ such that

(1) $\psi_{1}$ is bilipschitzian with respect to the spherical metric in $\bar{R}^{3}$,

(2) the part of $\partial D$ lying in $H_{+}^{3}=\left\{x \in R^{3} \mid x_{3}>0\right\}$ is mapped onto $\partial D^{\prime} \cap H_{+}^{3}$,

(3) $\psi_{1} \mid \Delta$ is the identity. 
Next we perform a quasiconformal map $\psi_{2}: D^{\prime} \rightarrow \psi_{2} D^{\prime}$ by setting $\psi_{2}\left(\varrho, \varphi, x_{3}\right)=$ $\left(\varrho, 6 \varphi, x_{3}\right)$ in cylindrical coordinates in $D^{\prime} \backslash \Delta^{\prime \prime}$, where $\Delta^{\prime \prime}$ is the 3 -simplex concentric with $\Delta$ and with side length $3 a / 2$, and by requiring that $\psi_{2} \mid \Delta$ is the identity. Then $\psi_{3}=\psi_{2} \circ \psi_{1}$, when extended to $\bar{D}$ is a continuous map in the spherical metric and quasiconformal in $D$. The original constructions can be performed so that the map $w \circ \psi_{3}^{-1} \mid \psi_{3} W: \psi_{3} W \rightarrow$ int $\bar{U}_{3}$ is well defined also on $\overline{\psi_{3} W}$, particularly on the negative $x_{1}$-axis.

The complement of $\psi_{3} Y$ consists of three components, each homeomorphic to a 3-ball. One of them is $W_{0}=\psi_{3} W$. Let the others be $W_{+}$and $W_{-}$and let us fix the notation so that $W_{+}$is the one which contains the positive $x_{3}$-axis. On $\partial W_{+}$a subset of the set $\psi_{3}\left(\mathscr{H}_{k, k-1}^{2} \cup \hat{\mathscr{H}}_{k, k-1}^{2}\right)$ of sheets appears similarly as on $\partial W_{0}$, in particular, the numbers of sheets in $\partial W_{+}$and in $\partial W_{0}$ are equal. Apart from some metirical modifications we can repeat in $W_{+}$what we did in $W$ in Section 2 when we constructed the quasiregular map $w$. As a result we obtain a quasiregular map $w_{+}: W_{+} \rightarrow \operatorname{int} \bar{U}_{1}$ which coincides with $w_{0}=w \circ \psi_{3}^{-1}: W_{0} \rightarrow$ int $\bar{U}_{3}$ on common boundary parts. Furthermore, we can form $w_{+}$so that there exists a regular 3 -simplex $\Delta_{+}$, with the concentric 3-simplex $\Delta_{+}^{\prime}$ with double side length contained in $W_{+}$, and $w_{+} \mid \Delta_{+}$is the same as $w_{0}|\Delta=w| \Delta$ up to similarity maps. For $W_{-}$we obtain similarly $w_{-}, \Delta_{-}$and $\Delta_{-}^{\prime}$. This way we have defined a $K_{0}$-quasimeromorphic map $f_{0}: \bar{R}^{3} \rightarrow \bar{R}^{3}$ with the property $i\left(v, f_{0}\right)=i\left(v_{+}, f_{0}\right)=i\left(v_{-}, f_{0}\right)=\mu\left(f_{0}\right)$. Here $v_{+}$and $v_{-}$are the centers of $\Delta_{+}$ and $\Delta_{-}$. The construction can be made so that $K_{0}$ is an absolute constant, in particular, ît does not depend on $k$. But $\sigma(G) / 2=i\left(v, f_{0}\right)$ depends on $k$ and tends to infinity as $k \rightarrow \infty$. We still modify $f_{0}$ a little. We perform a quasiconformal map $\psi_{4}$ : $\bar{R}^{3} \rightarrow \bar{R}^{3}$, which is the identity outside $\Delta^{\prime} \cup \Delta_{+}^{\prime} \cup \Delta_{-}^{\prime}$, and maps each of the 3 -simplexes $\Delta^{\prime}, \Delta_{+}^{\prime}$, and $\Delta_{-}^{\prime}$ radially with respect to the centers such that $\Delta, \Delta_{+}$, and $\Delta_{-}$are mapped onto some balls $B_{3}=\bar{B}^{3}\left(v, r_{3}\right), B_{1}=\bar{B}^{3}\left(v_{+}, r_{1}\right)$, and $B_{2}=\bar{B}^{3}\left(v_{-}, r_{2}\right)$. The map $f_{1}=f_{0} \circ \psi_{4}^{-1}$ can be made $K$-quasimeromorphic with $K$ an absolute constant.

Let $\varphi_{j}$ be the inversion in $\partial B_{j}$ and $\varphi_{j}^{\prime}$ the inversion in $\partial f_{1} B_{j}, j=1,2,3$. In our next step we form the $K$-quasimeromorphic map $f_{2}: \bar{R}^{3} \rightarrow \bar{R}^{3}$ by setting

$$
\begin{gathered}
f_{2}\left|\bar{R}^{3} \backslash\left(B_{1} \cup B_{2} \cup B_{3}\right)=f_{1}\right| \bar{R}^{3} \backslash\left(B_{1} \cup B_{2} \cup B_{3}\right), \\
f_{2}\left|B_{j}=\varphi_{j}^{\prime} \circ f_{1} \circ \varphi_{j}\right| B_{j}, \quad j=1,2,3 .
\end{gathered}
$$

At the six points $\varphi_{1}(v), \varphi_{1}\left(v_{-}\right), \varphi_{2}(v), \varphi_{2}\left(v_{+}\right), \varphi_{3}\left(v_{+}\right), \varphi_{3}\left(v_{-}\right) f_{2}$ has local index $\mu\left(f_{2}\right)=\mu\left(f_{1}\right)=\mu\left(f_{0}\right)$. Repeating this for the six second generation balls $\varphi_{j} B_{i}, i \neq j$, $i, j=1,2,3$, and their images under $f_{2}$, we obtain a $K$-quasimeromorphic map $f_{3}$ with $5 \cdot 6=30$ points $x_{3,1}, \ldots, x_{3, j_{3}}, j_{3}=30$, with local index equal to $\mu\left(f_{3}\right)=\mu\left(f_{0}\right)$. This way we obtain a sequence $f_{1}, f_{2}, \ldots$ of $K$-quasimeromorphic maps of $\bar{R}^{3}$ onto itself which converges uniformly to a $K$-quasimeromorphic map $h: \bar{R}^{3} \rightarrow \bar{R}^{3}$. The degree $\mu(h)$ is $\mu\left(f_{0}\right)$ which is checked by looking at $h^{-1}(y)$ for some $y$ outside the balls $f_{1} B_{j}, j=1,2,3$.

According to the construction the set $E$ of accumulation points of the set 
$\left\{x_{i, j} \mid 1 \leqq j \leqq j_{i}, i=1,2, \ldots\right\}$ is a Cantor set. It remains to show that for each point $x \in E i(x, h)=\mu(h)$. Let $x \in E$. It is enough to show that $h^{-1}(h(x))=\{x\}$. Suppose $z \neq x$ and $h(z)=h(x)$. Since $x \in E$, there are balls of arbitrary high generation containing $x$. We can therefore find such a ball $V \ni x$ with $z \notin V$. But according to the construction $h\left(\bar{R}^{3} \backslash V\right) \cap h V=\emptyset$, which gives a contradiction with $h(z)=h(x)$. The theorem is proved.

\section{References}

[1] Martio, O.: A capacity inequality for quasiregular mappings. - Ann. Acad. Sci. Fenn. Ser. A I Math. 474, 1970, 1-18.

[2] Martio, O., S. Rickman, and J. VäIsÄLÄ: Definitions for quasiregular mappings. - Ann. Acad. Sci. Fenn. Ser. A I Math. 448, 1969, 1-40.

[3] MARTio, O., S. RickMAN, and J. VÄISÄLÄ: Topological and metric properties of quasiregular mappings. - Ann. Acad. Sci. Fenn. Ser. A I Math. 488, 1971, 1-31.

[4] Rickman, S.: A defect relation for quasimeromorphic mappings. - Ann. of Math. (2) 114, 1981, 165-191.

[5] Rickman, S.: The analogue of Picard's theorem for quasiregular mappings in dimension three. Acta Math. 154, 1985, 195-242.

[6] Rickman, S., and U. Srebro: Remarks on the local index of quasiregular mappings. - To appear.

[7] Zorič, V. A.: M. A. Lavrent'ev's theorem on quasiconformal space maps. - Mat. Sb. (N. S.) 74, 1967, 417-433 (Russian).

University of Helsinki

Department of Mathematics

SF-00100 Helsinki

Finland

Received 2 April 1984 